\title{
The geodesic structure of the Schwarzschild Anti-de Sitter black hole
}

\author{
Norman Cruz * $^{7}$ Marco Olivares 7 and J.R.Villanuevd \\ Departamento de Física, Facultad de Ciencia, Universidad de Santiago de Chile, Casilla 307, Santiago 2, Chile
}

(Dated: February 3, 2008)

\begin{abstract}
In the present work we found the geodesic structure of an AdS black hole. By means of a detailed analyze of the corresponding effective potentials for particles and photon, we found all the possible motions which are allowed by the energy levels. Radial and non radial trajectories were exactly evaluated for both geodesics. The founded orbits were plotted in order to have a direct visualization of the allowed motions. We show that the geodesic structure of this black hole presents new type of motions not allowed by the Schwarzschild spacetime.
\end{abstract}

\section{INTRODUCTION}

The presence of a vacuum energy (cosmological constant) has been considered in theoretical models related to unification as superstring and to cosmology and astrophysics. A possitive cosmological constant is supported by the measurements of distant SNe Ia (at $z \sim 1$ ), which indicate that the expansion of the Universe is in accelaration 1]. Other tests of the standard model, including spacetime geometry, galaxy peculiar velocities, structure formation, and early Universe physics, supports, in many of these cases, a flat Universe model with the presence of a cosmological constant [2].

Of course, the above evidences have motivated to consider spherical symmetric spacetimes with non zero vacuum energy in order to study the well known effects predicted by General Relativity for planetary orbits and massless particles. This study implies to determine the geodesic structure of Kottler spacetimes [3]. Timelike geodesics for positive cosmological constant were investigated in [4], using only the method of an effective potential in order to found the conditions for the existence of bound orbits. The analyze of the effective potential for radial null geodesic in Reissner-Nordstrom-de Sitter and Kerr-de Sitter spacetime was realized in [5]. Podolsky 6] investigated all possible geodesic motions for extreme Schwarzschild-de Sitter spacetime.

Classical test of general relativity such as the bending of light was examined by Lake [17], founding that the cosmological constant produces no change in this effect. Exact solutions in closed analytic form for geodesic motion in Kottler spacetime were found very recently by Kraniotis et al [7]. The exact solutions of the timelike geodesic were used to evaluate the perihelion precession of the planet Mercury. The motion of massive particles in the Kerr and Kerr-(anti) de Sitter gravitational fields was investigated in [8], where the geodesic equations are derived, by solving the Hamilton-Jacobi partial differential equation.

The main purpose of this article is to find exact solutions for time-like and null geodesic of a Schwarzschild Anti-de Sitter spacetime. We have restricted to this spacetime since our objectives are: i) to investigate by means of a detailed analyze of the effective potentials, all the possible movements which are allow by the energy levels, ii) to find the exact solutions describing the trajectories of massive and null particles, and iii) to plot the founded orbits in order to have a direct visualization of the allowed motions.

In section 2, we derive the geodesic equations of motion using the variational problem associated to this metric. We find the equivalent one dimensional problem with the respective effective potential.

In section 3 the effective potential is analyzed in order to determine the possible motions of massive particles. The exact solutions are calculate for the allowed motions. In particular, we show the exact solution in the proper time for a radial geodesic. Planetary orbits are integrated to obtain the polar equation in term of incomplete integrals of Jacobi. Using an elementary derivation the advance of perihelion is evaluated. We also gives an exact solution for planetary orbits which could end into the event horizon in terms of elementary functions.

In section 4 null geodesics are analyzed in terms of the one dimensional effective potential. Explicit exact solutions are found for radial geodesics in terms of the proper and coordinate times. Bounded and unbounded geodesics are integrated in terms of Jacobian elliptic integrals. The bounded geodesics do not exist in the Schwarzschild spacetime.

In section 5 we summarize and discuss the results founded.

\section{GEODESICS}

*Electronic address: ncruz@lauca.usach.cl

${ }^{\dagger}$ Electronic address: marcofisica@gacg.cl

‡Electronic address: jrvillanueva@gacg.cl
The metric for a static spherically symmetric spacetime with mass $M$ and a negative cosmological constant $\Lambda=$ 
$-3 / \ell^{2}$ is

$$
d s^{2}=-f(r) d t^{2}+\frac{d r^{2}}{f(r)}+r^{2}\left(d \theta^{2}+\sin ^{2} \theta d \phi^{2}\right),
$$

where the lapse function, $f(r)$, is given by

$$
f(r)=1-\frac{2 M}{r}+\frac{r^{2}}{\ell^{2}},
$$

and the coordinates are defined such that $-\infty \leq t \leq$ $+\infty, r \geq 0,0 \leq \theta \leq \pi$ and $0 \leq \phi \leq 2 \pi$.

The lapse function vanished at the zeros of the cubic equation

$$
r^{3}+\ell^{2} r-2 M \ell^{2}=0
$$

The only real root of this equation is (see appendix A for more details)

$$
r_{+}=\frac{2}{3} \sqrt{3} \ell \sinh \left[\frac{1}{3} \sinh ^{-1}\left(3 \sqrt{3} \frac{M}{\ell}\right)\right]
$$

Expanding $r_{+}$in term of $M$ with $1 / \ell^{2} \ll M^{2} / 9$, we obtain

$$
r_{+} \approx 2 M-\frac{8 M^{3}}{\ell^{2}}+\ldots
$$

The event horizon of the $A d S$ black hole is lower than the Schwarzschild event horizon, $r_{+S c h}=2 M$.

In order to find the geodesics structure of the spacetime described by (11), we solve the Euler-Lagrange equations for the variational problem associated to this metric (see [9], for instance). The corresponding Lagrangian is

$$
\mathcal{L}=-f(r) \dot{t}^{2}+f^{-1}(r) \dot{r}^{2}+r^{2}\left(\dot{\theta}^{2}+\sin ^{2} \theta \dot{\phi}\right),
$$

where the dots represents the derivative with respect to the affine parameter $\tau$, along the geodesic.

The equations of motion are

$$
\dot{\Pi}_{q}-\frac{\partial \mathcal{L}}{\partial q}=0
$$

where $\Pi_{q}=\partial \mathcal{L} / \partial \dot{q}$ is the momentum conjugate to coordinate $q$.

Since the Lagrangian is independent of $(t, \phi)$ the corresponding conjugate momenta are conserved, therefore

$$
\Pi_{t}=-\left(1-\frac{2 M}{r}+\frac{r^{2}}{\ell^{2}}\right) \dot{t}=-E,
$$

and

$$
\Pi_{\phi}=r^{2} \sin ^{2} \theta \dot{\phi}=L
$$

where $E$ and $L$ are constant of motion.

Now, from the equation of motion for $\theta$, we have

$$
\frac{d\left(r^{2} \dot{\theta}\right)}{d \tau}=r^{2} \sin \theta \cos \theta \dot{\phi}^{2}, .
$$

For simplicity, let us choose the initial condition $\theta=\pi / 2$ and $\dot{\theta}=0$. Then, from the last equation we find that $\ddot{\theta}=0$. This means that the motion is confined to the plane $\theta=\pi / 2$, which is characteristic of the central fields. With this election equation (9) becomes

$$
\Pi_{\phi}=r^{2} \dot{\phi}=L
$$

and, from (8) and (11), the Lagrangian (6) can be write in the following form

$$
2 \mathcal{L} \equiv h=\frac{E^{2}}{1-\frac{2 M}{r}+\frac{r^{2}}{\ell^{2}}}-\frac{\dot{r}^{2}}{1-\frac{2 M}{r}+\frac{r^{2}}{\ell^{2}}}-\frac{L^{2}}{r^{2}} .
$$

By normalization, we shall consider that $h=1$ for massive particles and $h=0$ for photons. We solve the above equation for $\dot{r}^{2}$ in order to obtain the radial equation, which allow us to characterize possible moments of test particles without and explicit solution of the equation of motion in the invariant plane

$$
\dot{r}^{2}=E^{2}-\left(1-\frac{2 M}{r}+\frac{r^{2}}{\ell^{2}}\right)\left(h+\frac{L^{2}}{r^{2}}\right) .
$$

It is useful to rewrite (13) as the equation of motion of a one dimensional problem

$$
\dot{r}^{2}=E^{2}-V_{e f f}^{2} .
$$

where $V_{e f f}^{2}$ define an effective potential

$$
V_{e f f}^{2}=\left(1-\frac{2 M}{r}+\frac{r^{2}}{\ell^{2}}\right)\left(h+\frac{L^{2}}{r^{2}}\right),
$$

\section{TIME-LIKE GEODESICS}

For time-like geodesic $h=1$ and the effective potential becomes

$$
V_{e f f}^{2}=\left(1-\frac{2 M}{r}+\frac{r^{2}}{\ell^{2}}\right)\left(1+\frac{L^{2}}{r^{2}}\right) .
$$

Using this effective potential, we solve the radial equation (13) for radial and non-radial particles. 


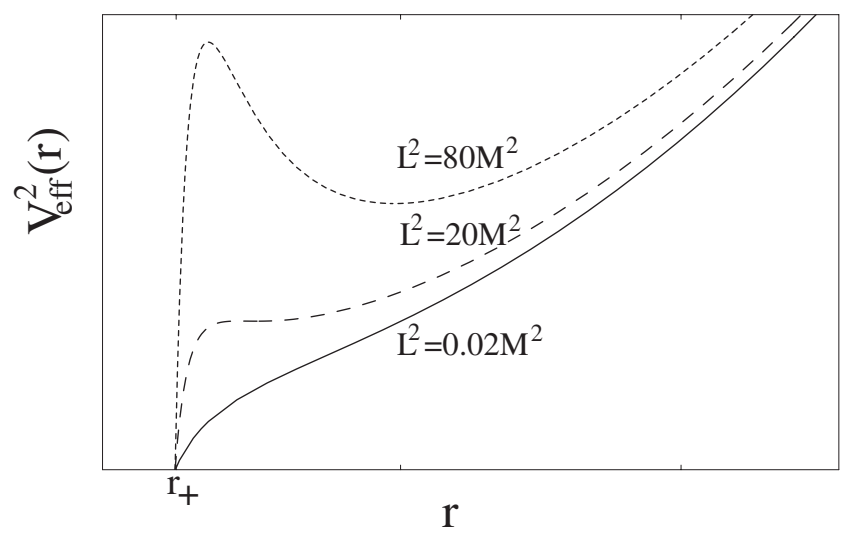

FIG. 1: The figure shows the evolution of the effective potential for non-radial particles at different values of the constant of motion $L$.

\section{A. Radial geodesics}

The radial geodesic correspond to the motion of particles without angular momentum $L=0$, which are falling from rest, in a finite distance, to the center. In this case, the effective potential becomes

$$
V_{e f f}^{2}=\left(1-\frac{2 M}{r}+\frac{r^{2}}{\ell^{2}}\right) .
$$

which is showed in FIG 2 Therefore, the radial equation is

$$
\dot{r}^{2}=E^{2}-\left(1-\frac{2 M}{r}+\frac{r^{2}}{\ell^{2}}\right) .
$$

In the elliptic curve representing $V_{e f f}^{2}$ particles always plunges into the horizon from an upper distance determined by the constant of motion $E$. This fact is due to attractive force generated by $\Lambda<0$. In order to find exact solutions we solve, by simplicity, the case when two of the roots of the cubic equation are equals [19]. The corresponding energy is $E_{0}^{2}=1+3 \sqrt[3]{\frac{M^{2}}{\ell^{2}}}$. For this value of $E$, we obtain that $r_{0}=2 \sqrt[3]{M \ell^{2}}$. The constant of motion $E$ in terms of $r_{0}$ yields

$$
E_{0}^{2}=1+\frac{3}{4 \ell^{2}} r_{0}^{2}
$$

Replacing this values in the radial equation (18), we obtain

$$
-\frac{d r}{d \tau}=\frac{1}{\ell}\left(r+\frac{r_{0}}{2}\right) \sqrt{\frac{r_{0}-r}{r}}
$$

Making the change of variable $r=r_{0} \cos ^{2} \eta / 2$, the above equation yields

$$
\frac{d \eta}{d \tau}=\frac{1}{\ell} \frac{2+\cos \eta}{1+\cos \eta}
$$

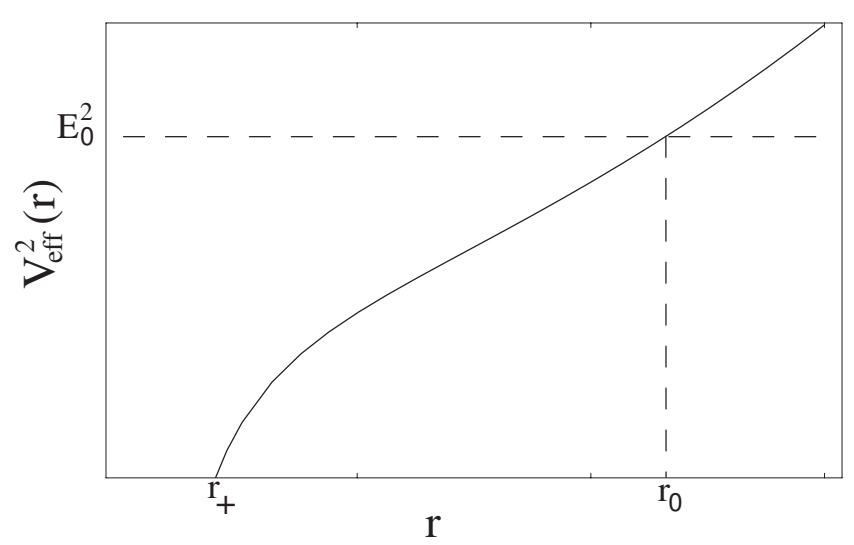

FIG. 2: The figure shows the effective potential for radial particles.

A forward integration of this last equation yields

$$
\tau(\eta)=2 \ell\left[\frac{\eta}{2}+\frac{\sqrt{3}}{3} \arctan \frac{\sqrt{3}}{3} \tan \left(\frac{\eta}{2}\right)\right]
$$

In term of the variable $r,\left(\eta_{0}=0\right.$ if $r=r_{0}$ and, from (22), $\left.\tau_{0}=0\right)$

$$
\tau(r)=2 \ell\left[\arccos \left(\frac{r}{r_{0}}\right)+\frac{\sqrt{3}}{3} \arctan \frac{\sqrt{3}}{3} \frac{\sqrt{r_{0}-r}}{r}\right]
$$

In FIG 3 equation (23) is plotted. The particle falls to the horizon in a finite proper time lower than in the Schwarzschild case.

Now, we shall see that the situation is very different when consider the equation of the trajectory in coordinate time $t$. The equation to be integrated in order to 


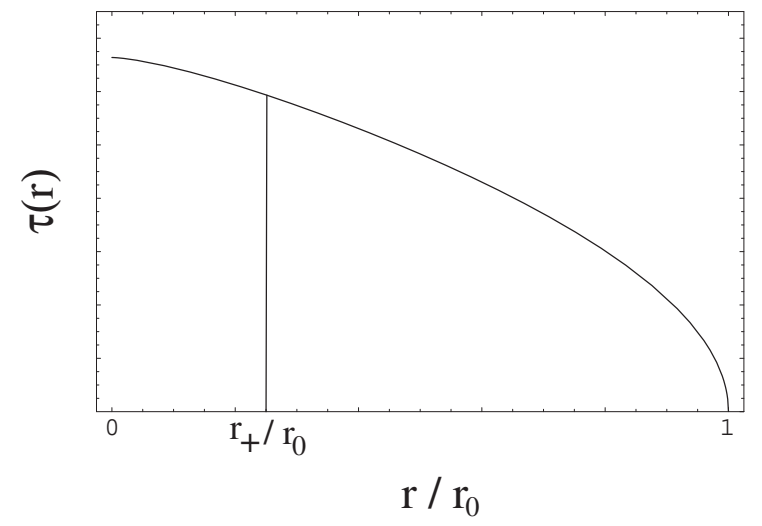

FIG. 3: The figure shows the proper time $\tau$ as a function of $r / r_{0}$.

obtain $t$ is (cf. equation (8) and (20)

$$
\frac{d t}{d r}=-\ell^{3} E \frac{r}{\left(r^{2}+r_{+} r+\rho^{2}\right)\left(r-r_{+}\right)\left(r+\frac{r_{0}}{2}\right) \sqrt{\frac{r_{0}-r}{r}}}
$$

Using the substitution $r=r_{0} \cos ^{2} \eta / 2$, this equation gives

$$
t(\eta)=A[B \tau(\eta)+C H(\eta)+D \eta-I(\eta)]
$$

where

$$
\begin{array}{ll}
A=\frac{\ell^{3} E}{r_{0}+2 r_{+}}, & B=\frac{r_{0}}{\rho^{2}+\frac{r_{0}^{2}}{4}-\frac{r_{0} r_{+}}{2}}, \\
C=\frac{2 r_{+}}{\rho^{2}+2 r_{+}}, & D=B+C,
\end{array}
$$

and

$$
\begin{gathered}
H(\eta)=\eta+\cot \frac{\eta_{+}}{2} \ln \left|\frac{\tan \frac{\eta_{+}}{2}+\tan \frac{\eta}{2}}{\tan \frac{\eta_{+}}{2}-\tan \frac{\eta}{2}}\right|, \\
I(\eta)=\int_{0}^{\eta} \frac{a \cos ^{2} \frac{\eta}{2}+b}{r_{0}^{2} \cos ^{4} \frac{\eta}{2}+r_{0} r_{+} \cos ^{2} \frac{\eta}{2}+\rho^{2}} d \eta .
\end{gathered}
$$

Also, $\tau(\eta)$, which is given by (22), together with the integral $I(\eta)$ are well defined when $\eta \rightarrow \eta_{+}$. Moreover, in these limit, the function $H(\eta)$ goes to infinity, which implies that the coordinate time, $t$, goes to infinity too. This physical result is in accord with the Schwarzschild case.

\section{B. The bound orbits}

In this case we have that $L \neq 0$, and the radial equation takes the form

$$
\dot{r}^{2}=E^{2}-\left(1-\frac{2 M}{r}+\frac{r^{2}}{\ell^{2}}\right)\left(1+\frac{L^{2}}{r^{2}}\right)
$$

In FIG 4 the effective potential has been plotted for nonradial particles when $L^{2}>11.25 M^{2}$. The following orbits are allowed depending on the value of the constant E

1.- If $E^{2}=E_{I}^{2}$, the particle can orbit in a stable circular orbit at $r=r_{c}$. The other possible orbit correspond to a particle at the point $B$ which plunges into the singularity.

2.- If $E_{I}^{2}<E^{2}<E_{I I}^{2}$, the particle orbit on a bound orbit in the range $r_{P}<r<r_{A}$, where $r_{P}$ and $r_{A}$ are the perihelion and aphelion distance, respectively. This orbits will be calling orbits of the first kind. The other possible orbit correspond to a particle at the point $D$ which plunges into the singularity.

3.- If $E^{2}=E_{I I}^{2}$, the particle can orbit in a unstable circular orbit at $r=F$. What is more likely is that a particle is such type of orbit will recede from $r=F$ to either $r=G$ or the singularity at $r=0$.

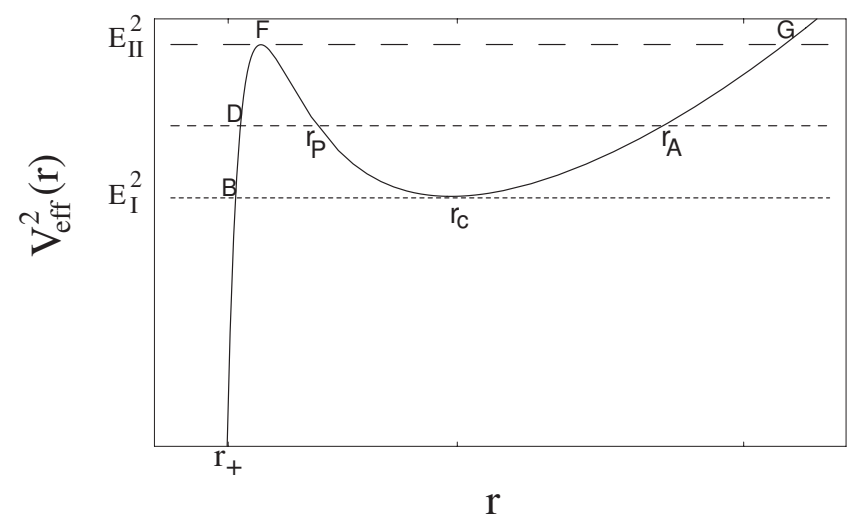

FIG. 4: The figure shows the effective potential for non-radial particles when $L^{2}>11.25 M^{2}$. The horizontal lines represent different labels of the constant of motion $E^{2}$.

Is possible to obtain the orbits equations using equation (11) in (29) and making the change of variable $u=r^{-1}$, we obtain

$$
\left(u \frac{d u}{d \phi}\right)^{2}=2 M P(u)
$$

where the polynomial $P(u)$ is given by

$$
P(u)=u^{5}-\frac{1}{2 M} u^{4}+\frac{1}{L^{2}} u^{3}+\frac{\varepsilon^{2}}{2 M L^{2}} u^{2}-\frac{1}{2 M L^{2} \ell^{2}}
$$


and the parameter $\varepsilon^{2}$ is defined by the relation

$$
\varepsilon^{2} \equiv E^{2}-1-\frac{L^{2}}{\ell^{2}} \equiv E^{2}-E_{\Lambda}^{2} .
$$

As we see, from FIG 4 bound orbits can exist in an Schwarzschild AdS space-time. This fact is imposed by the condition $\varepsilon^{2}>0$.

Since $P(u)$ is a 5 degrees polynomial, our analyze take an easy form if we consider that this polynomial posses two identical negative roots, $u_{4}=u_{5}=-\sigma$. Therefore, we have

$$
P(u)=(u+\sigma)^{2} g(u),
$$

where $g(u)$ is a cubic polynomial, which posses the information of the physical range of interest. This polynomial is the key for the study of the different kind of orbits that we shall pass to analyze. In term of $g(u)$, (30) is integrated, yielding

$$
\pm \Delta \phi=\frac{1}{\sqrt{2 M}} \int_{u_{0}}^{u} \frac{d u^{\prime}}{\sqrt{g\left(u^{\prime}\right)}}-\frac{\sigma}{\sqrt{2 M}} \int_{u_{0}}^{u} \frac{d u^{\prime}}{\left(u^{\prime}+\sigma\right) \sqrt{g\left(u^{\prime}\right)}} .
$$

\section{Orbits Of The First Kind}

The orbits of the first kind represents bound geodesics, with two extreme values. These type of orbits are allowed when $E$ and $L$ satisfy $E<1$ and $L^{2}>11.25 M^{2}$. We can identify 3 class of orbits of the first kind: Planetary orbits, asymptotic orbits and circular orbits.

\section{(i)Planetary orbits}

The planetary orbits are constrain to oscillate between an aphelion and a perihelion. These distances correspond to the roots of $g(u)$, therefore, the another root is also real and positive, which shall must correspond to the aphelion distance at the continuation of the planetary orbit. In order to obtain these root explicitly, we make the substitution

$$
u=\frac{1+e \cos \chi}{R}
$$

where $e$ represent the eccentricity of the orbits, and $R$ is the latus rectum. Therefore, solving the equations $B$ B (B10), we find

$$
u_{1}=\frac{1-e}{R}, \quad u_{2}=\frac{1+e}{R}, \quad u_{3}=\frac{1}{2 M}-\frac{2}{R}+2 \sigma .
$$

where $u_{1}$ correspond to the inverse of the aphelion distance $(\chi=\pi), u_{2}$ is the inverse of the perihelion distance $(\chi=0)$ and $u_{3}$ is the inverse of an aphelion distance for the orbits of second kind, which we shall study in the next section. The physical range in this case is $r>r_{3}$ (i.e, $u_{3}>u$ ). Therefore, the cubic polynomial $g(u)$ can be rewrite as $g(u)=\left(u-u_{1}\right)\left(u-u_{2}\right)\left(u_{3}-u\right)$. Thus, the equation (34) becomes

$$
\pm \Delta \phi=\frac{1}{A} \int_{\pi}^{\chi} \frac{-d \chi}{\sqrt{1-k^{2} \sin ^{2}\left(\frac{\pi}{2}-\frac{\chi}{2}\right)}}-\frac{\nu n^{2}}{2 e A} \int_{\pi}^{\chi} \frac{-d \chi}{\left(1+n^{2} \sin ^{2}\left(\frac{\pi}{2}-\frac{\chi}{2}\right)\right) \sqrt{1-k^{2} \sin ^{2}\left(\frac{\pi}{2}-\frac{\chi}{2}\right)}} .
$$

where

$$
\begin{gathered}
A^{2}=1-6 \mu+2 \mu e+4 \mu \nu, \\
k^{2}=\frac{4 \mu e}{A^{2}},
\end{gathered}
$$

$$
\mu=\frac{M}{R} \quad \nu=\sigma R
$$


In term of incomplete integrals of Jacobi, the above solution can be rewrite as

$$
\phi=\frac{2}{A}\left[F\left(k, \frac{\pi}{2}-\frac{\chi}{2}\right)-\frac{\nu n^{2}}{2 e} \Pi\left(n, k, \frac{\pi}{2}-\frac{\chi}{2}\right)\right] .
$$

(i.1)Advance of the perihelion.

We use the elementary derivation of the advance of perihelion of a planetary orbit given in 12] for the Schwarzschild solution. In our case, the advance of the perihelion is obtain comparing a keplerian ellipse in a Lorentzian coordinates with one in a Schwarzschild Antide Sitter coordinates. The relevant relation communicating the two ellipse is the areal constant of Kepler's second law. In unperturbed Lorentz coordinates the line element is given by

$$
d s^{2}=-d t^{2}+d r^{2}+r^{2}\left(d \theta^{2}+\sin ^{2} \theta d \phi^{2}\right) .
$$

The Schwarzschild Anti-de Sitter (SAdS) gravitational field, given by equation (2), allow to find the following transformation of the coordinates, $r$ and $t$, in the binomial approximation

$$
\begin{aligned}
& d t^{\prime}=\left(1-\frac{M}{r}+\frac{r^{2}}{2 \ell^{2}}\right) d t, \\
& d r^{\prime}=\left(1+\frac{M}{r}-\frac{r^{2}}{2 \ell^{2}}\right) d r .
\end{aligned}
$$

We consider two elliptical orbits, one the classical Kepler orbit in $r, t$ space and a Schwarzschild Anti-de Sitter orbit in an $r^{\prime}, t^{\prime}$ space. In the Lorentz space we have

$$
d A=\int_{0}^{\rho} r d r d \phi
$$

and hence the Kepler second law

$$
\frac{d A}{d t}=\frac{1}{2} \rho^{2} \frac{d \phi}{d t} .
$$

In the Schwarzschild Anti-de Sitter situation however, we have

$$
d A^{\prime}=\int_{0}^{\rho} r d r^{\prime} d \phi
$$

with $d r^{\prime}$ given by equation (45). Therefore, applied wherever necessary the binomial approximation and the transformation of coordinate, we obtain

$$
\frac{d A^{\prime}}{d t^{\prime}}=\frac{\rho^{2}}{2}\left(1+\frac{3 M}{\rho}+\frac{2 M^{2}}{\rho^{2}}-\frac{3 \rho^{2}}{4 \ell^{2}}-\frac{5 M \rho}{4 \ell^{2}}+\ldots\right) \frac{d \phi}{d t} .
$$

Applying all of this increasing for a single orbit

$$
\Delta \phi^{\prime}=\int_{0}^{2 \pi}\left(1+\frac{3 M}{\rho}+\frac{2 M^{2}}{\rho^{2}}-\frac{3 \rho^{2}}{4 \ell^{2}}-\frac{5 M \rho}{4 \ell^{2}}+\ldots\right) d \phi
$$

For an ellipse $\rho=\frac{R}{1+e \cos \phi}$ where $e$ is the eccentricity and $R$ is the latus rectum. Therefore, applying the binomial approximation, we obtain

$$
\Delta \phi^{\prime} \approx 2 \pi+\frac{6 \pi M}{R}+\frac{4 \pi M^{2}}{R^{2}}-\frac{3 \pi R^{2}}{2 \ell^{2}}-\frac{5 \pi M R}{2 \ell^{2}}+\ldots
$$

the classical advance of perihelion is recuperated for zero cosmological constant $(\ell \rightarrow \infty)$. The two last terms are the corrections due to a negative cosmological constant.

\section{(ii)Asymptotic orbits}

This case occur when two real roots of $g(u)$ are coincident and positive, namely $u_{2}=u_{3}$, and also this roots are placed in the extremal of the effective potential, such that the energy is characterized by $E_{I I}^{2}$. The substitution is

$$
u=\frac{1+e \cos \chi}{R} .
$$

therefore, we have that

$$
u_{1}=\frac{1-e}{R} \quad \text { and } \quad u_{2}=u_{3}=\frac{1+e}{R},
$$

are the inverse of the aphelion and the perihelion distance, respectively. Is possible to solve the roots equation to obtain the exact value of $u_{4} u_{5}=-\sigma$ (See appendix B for more details), resulting

$$
\sigma=\frac{2}{R} \frac{1-e^{2}}{3-e}
$$

With this substitution, the equation (34) can be integrated in terms of elementary functions. Assuming that the particles are falling from the aphelion distance, where we choose $\phi=0$, we obtain 


$$
\phi=\frac{\sqrt{2\left(5+3 e^{2}\right)(3-e)}}{5-3 e}\left[-\ln \left(\tan \frac{\chi}{4}\right)+\sqrt{\frac{8 e(1-e)}{(5+e)(3-e)}} \arctan \sqrt{\frac{e(3-e)(1+\cos \chi)}{(5+e)(1-e)}}\right]
$$

The second function on the right hand side correspond to the cosmological correction of the Schwarzschild solution founded by [11].

\section{(iii) Circular orbits}

In this case, we have that $r=r_{c}=C t e$, where $r_{c}$ correspond to the distance of the circular orbit from the singularity. Is possible calculate the periods of these orbit making use of the constant of motion $E$ y $L$, given by (8) and (11) respectively. In fact, dividing between them, we find

$$
d t=\frac{E}{L} \frac{r_{c}}{f\left(r_{c}\right)} d \phi
$$

The circular orbit condition, $\left(V_{e f f}^{2}\right)^{\prime}=0$, give us a five degrees equation in the variable $r$, moreover, this equation can be solved for $L$ in term of $r_{c}$, becomes

$$
L=\sqrt{\frac{r_{c}^{5}+\ell^{2} M r_{c}^{2}}{\ell^{2}\left(r_{c}-3 M\right)}}
$$

and also, for this orbits $E^{2}=V_{e f f}^{2}\left(r=r_{c}\right)$. In a period, $\Delta t \equiv T_{t}$, we have $\Delta \phi=2 \pi$. Thus, replacing this in (56), we obtain

$$
T_{t}=\frac{2 \pi \sqrt{\frac{r_{c}^{3}}{M}}}{\sqrt{1+\frac{r_{c}^{3}}{\ell^{2} M}}} \equiv \frac{T_{t, S c h}}{\sqrt{1+\frac{r_{c}^{3}}{\ell^{2} M}}}
$$

where $T_{t, S c h}$ is the period in the coordinate time founded for the Schwarzschild spacetime [10]. In the same way, for the proper time, using equation (11), yields

$$
T_{\tau}=\frac{2 \pi r_{c} \sqrt{\frac{r_{c}}{M}-3}}{\sqrt{1+\frac{r_{c}^{3}}{\ell^{2} M}}} \equiv \frac{T_{\tau, S c h}}{\sqrt{1+\frac{r_{c}^{3}}{\ell^{2} M}}}
$$

where $T_{\tau, S c h}$ is the period in the proper time founded for the Schwarzschild spacetime [10].

These period are lower than the corresponding to the Schwarzschild orbits, which is consistent with fact a negative cosmological constant increase the gravitational attraction.

\section{Orbits Of The Second Kind}

These type of orbits are allowed when $E$ and $L$ satisfy $E<1$ and $L^{2}>12 M^{2}$, which are the same conditions of the orbits of the first kind. Nevertheless, these orbits represents allowed motions in the regions at the left hand side of the top of the effective potential. Particles following these orbits fall to the event horizon.

By simplicity we shall denote with the same name the second kind trajectories as long as they have the same energy as the orbits of the first kind.

\section{(i) Planetary orbits}

The planetary orbits of the second kind, posses the same energy that the planetary orbits of the first kind, moreover these are starting from an aphelion distance $r=r_{3}=A$, which is greater than the variable $r$. This mean that the physical range is now $r<A$ or $u>u_{3}$. Therefore, the cubic polynomial $g(u)$ must be write as $g(u)=\left(u-u_{1}\right)\left(u-u_{2}\right)\left(u-u_{3}\right)$, and the substitution is

$$
u=u_{3}+\left(u_{3}-u_{2}\right) \tan ^{2}\left(\frac{1}{2} \xi\right)
$$

where $u_{1}, u_{2}$ and $u_{3}$ are given by equation (36). For this substitution

$$
\begin{array}{rrrr} 
& u=u_{3} & \text { for } & \xi=0, \\
u \rightarrow \infty \quad \text { and } \quad r \rightarrow 0 & \text { for } & \xi=\pi .
\end{array}
$$

Moreover, the form of the solution is the same that in the first kind counterpart (42). Thus

$$
\phi=\frac{2}{A}\left(1-\frac{\nu}{\mu+\nu}\right) F(\xi / 2, k)+\frac{2 \nu A}{(\mu+\nu) b} \Pi(\xi / 2, k, n),
$$

where

$$
b=1-4 \mu+6 \nu+2 \mu e .
$$

$$
n^{2}=\frac{2(\mu+\nu)}{b} .
$$

and $A, k$ are given by (38) and (39), respectively.

\section{(ii) Asymptotic orbits}


This case correspond to the continuation of the asymptotic orbit described above, but now the physical range is $u>u_{2}=u_{3}$. Therefore, we make the substitution

$$
u=\frac{1+e+2 e \tan ^{2} \frac{1}{2} \xi}{R},
$$

whit the same value of the roots (53) and (54). Then, after a little manipulation, we obtain

$$
\phi=\frac{\sqrt{2\left(5+3 e^{2}\right)(3-e)}}{5-3 e}\left[-\ln \left(\tan \frac{\chi}{4}\right)+\sqrt{\frac{8 e(1-e)}{(5+e)(3-e)}} \arctan \sqrt{\frac{e(3-e) \sec ^{2} \frac{1}{2} \xi}{(5+e)(1-e)}}\right] .
$$

In same way that in the asymptotic orbit of the first kind, the second function on the right hand side correspond to the cosmological correction to the Schwarzschild solution.

\section{NULL GEODESICS}

For photons $h=0$, and the effective potential given in (10) becomes

$$
V_{e f f}^{2}=\left(1-\frac{2 M}{r}+\frac{r^{2}}{\ell^{2}}\right) \frac{L^{2}}{r^{2}}
$$

In FIG 5 this effective potential has been plotted for non-radial photons. For different values of the constant $E$ the allowed orbits are as follows

1.- If $E^{2} \leq E_{\Lambda}^{2}-1$, photons plunges into the singularity from initial distances $r \leq 2 M$. For $E^{2}=E_{\Lambda}^{2}-1$ photons orbits are cardioid (see $I V .2$ ). This orbits do not exist in the Schwarzschild case. In the subsection B we will refer to these orbits as bound orbits.

The following geodesics are unbounded. They are explicitly integrated in subsection $\mathrm{C}$.

2.- If $E_{\Lambda}^{2}-1 \leq E^{2} \leq E_{C}^{2}$, photons can fall from the infinity to a minimum distance $r=P$ and them comes back to the infinity. The photons is only deflected. The other allowed orbits correspond to photons moving in the other side of the potential barrier, which plunges into the singularity.

3.- If $E^{2}=E_{C}^{2}$, the photons can orbit in an unstable circular orbit at $r=3 M$. The radius of this orbit is independent of $L ; L$ only affects the value of the energy $E_{C}^{2}$. Photons from the infinity can fall moving asymptotically to the circle $r=3 M$.

4.- If $E^{2}>E_{C}^{2}$, photons from the infinity plunges into the singularity.
Therefore, the radial equation in this section is given by

$$
\left(\frac{d r}{d \tau}\right)^{2}=E^{2}-\left(1-\frac{2 M}{r}+\frac{r^{2}}{\ell^{2}}\right) \frac{L^{2}}{r^{2}}
$$

and the effective potential is sketched in FIG 5

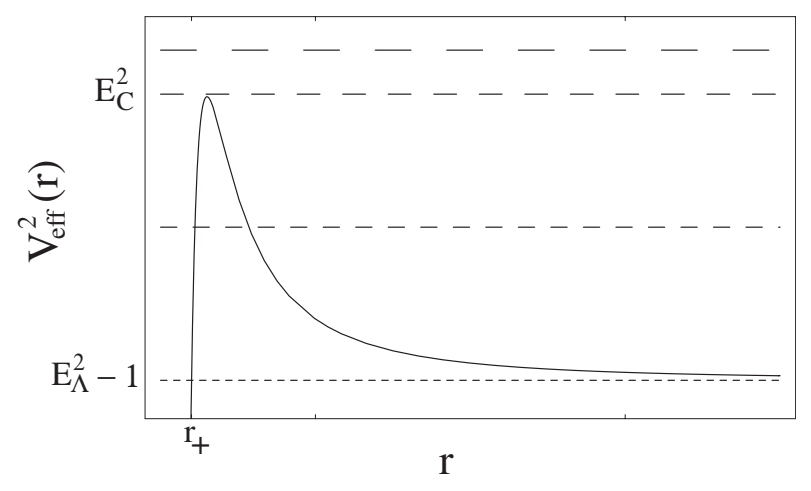

FIG. 5: The figure shows the typical effective potential for non-radial photons. The different level of energy are showed.

Due to the existence of a negative cosmological constant, the event horizon, $r_{+}$, is less than $2 M$, which value correspond to the event horizon in the Schwarzschild case. Photons falling from a distance $r_{0}=2 M$, posses a critical energy equal to $E_{\Lambda}^{2}-1$. Differentiating (68) with respect to the affin parameter, $\tau$, we found a pseudo Newton's law

$$
\ddot{r}=-\frac{1}{2}\left(V_{e f f}^{2}\right)^{\prime}
$$


The radial acceleration posses a maximum at $r=4 M$, this result is independent of the cosmological constant. Thus, photons with energy $E^{2}=E_{C}^{2}$ will posses a maximal radial acceleration, and photons in a intestable circularly orbits $\left(\mathrm{r}=3 \mathrm{M}\right.$, and energy $\left.E_{C}^{2}\right)$ will posses a zero radial acceleration.

On the other side, making the classical change of variable $u=1 / r$, together with (11), the radial equation (68) can be reduced to

$$
\left(\frac{d u}{d \phi}\right)^{2}=2 M u^{3}-u^{2}+\frac{1}{D^{2}}=z(u) \quad(\text { say }),
$$

where

$$
\frac{1}{D^{2}}=\frac{E^{2}}{L^{2}}-\frac{1}{\ell^{2}}
$$

denotes the impact parameter. In this case, the impact parameter is more less than its Schwarzschild counterpart, given by $\frac{E^{2}}{L^{2}}$.

\section{A. Radial geodesics}

In this case, $L=0$, which implies that the radial equation adopt the simple form

$$
\frac{d r}{d \tau}= \pm E
$$

where $(+)$ is for outgoing photons and $(-)$ is for ingoing photons. As in the Schwarzschild case, evaluated in [1]], the integration of the above equation yields

$$
r= \pm E \tau+r_{0}
$$

where $r_{0}$ is a constant of integration corresponding to the initial position of the photon. This result shows that for photons, in terms of the proper time, the radial motion is independent of the cosmological constant.

In order to obtain the equation of motion in terms of the coordinate time $t$, we makes use of the equations (8) and (72), yielding

$$
\frac{d r}{d t}= \pm\left(1-\frac{2 M}{r}+\frac{r^{2}}{\ell^{2}}\right) .
$$

or,

$$
\frac{d r}{d t}= \pm \frac{1}{\ell^{2} r}\left(r-r_{+}\right)\left(r^{2}+r_{+} r+\rho^{2}\right) .
$$

The solution is given by

$$
t= \pm \frac{\ell^{2} r_{+}}{2 r_{+}+\rho^{2}}\left[\ln \left(r-r_{+}\right)-\frac{1}{2} \ln \left(r^{2}+r r_{+}+\rho^{2}\right)+\delta \arctan \left(\frac{2 r+r_{+}}{\beta}\right)\right]+B,
$$

where $\alpha=\frac{\rho^{2}}{r_{+}}, \beta=\sqrt{4 \rho^{2}-r_{+}^{2}}, \delta=\frac{2 \alpha+r_{+}}{\beta}, \rho^{2}=\frac{2 \ell^{2} M}{r_{+}}$ and $B$ is a integrating constant.

\section{B. The Bound Geodesics}

\section{The orbits with imaginary eccentricities}

These type of orbits can exist when the energy is such that $0<E^{2}<L^{2} / \ell^{2} \equiv E_{\Lambda}^{2}-1$. Defining a new parameter $B$ by the expression

$$
\frac{1}{B^{2}}=\frac{1}{\ell^{2}}-\frac{E^{2}}{L^{2}}
$$

the radial equation becomes

$$
\left(\frac{d u}{d \phi}\right)^{2}=2 M u^{3}-u^{2}-\frac{1}{B^{2}}=2 M h(u)
$$

and the physical range is $r_{+}<r \leq r_{3}=A$, where $A$ is the aphelion distance for this orbits. The equation $h(u)=0$ allow only one real root, $A^{-1}$, and a complex conjugate pair, $u_{1}$ and $u_{2}$. For this reason, we shall characterize the roots of the $h(u)$ with a imaginary eccentricities

$$
u_{1}=\frac{-1+i e}{R}, \quad u_{2}=\frac{-1-i e}{R}, \quad u_{3}=\frac{1}{A} .
$$

The sum of the roots of the $h(u)$ allow us to calculate 
$u_{3}$

$$
u_{3}=\frac{1}{A}=\frac{1}{2 M}+\frac{2}{R}
$$

Thus, the second relation of the roots, $u_{1} u_{2}+u_{1} u_{3}+$ $u_{2} u_{3}=0$, yields

$$
\begin{aligned}
e^{2}-3=\frac{R}{M} & \equiv \frac{1}{\mu}>0, \\
& \Rightarrow e^{2}>3 .
\end{aligned}
$$

Finally, the product of roots, together with (81), becomes

$$
\left(\frac{R}{B}\right)^{2}=\frac{(1+4 \mu)^{2}}{\mu}
$$

In this way, the radial equation (78) is given by

$$
\left(\frac{d u}{d \phi}\right)^{2}=2 M\left[\left(u+\frac{1}{R}\right)^{2}+\frac{e^{2}}{R^{2}}\right]\left(u-\frac{1}{2 M}-\frac{2}{R}\right) .
$$

Using now the following substitution

$$
u=\frac{e \tan \frac{1}{2} \xi-1}{R} .
$$

Since the range of $u$ is

$$
\frac{1}{2 M}+\frac{2}{R} \leq u<\infty
$$

the corresponding range of $\xi$ is

$$
\xi_{0} \leq \xi<\pi
$$

where

$$
\tan \frac{1}{2} \xi_{0}=\frac{6 \mu+1}{2 \mu e}
$$

or, equivalently,

$$
\sin \frac{1}{2} \xi_{0}=\frac{6 \mu+1}{\triangle} \quad \text { and } \quad \cos \frac{1}{2} \xi_{0}=\frac{2 \mu e}{\triangle}
$$

where

$$
\triangle^{2}=(6 \mu+1)^{2}+4 \mu^{2} e^{2}
$$

We find that with the substitution (84), the equation (83) reduce to

$$
\frac{d \xi}{d \phi}= \pm \sqrt{2 \triangle} \sqrt{\sin \left(\xi-\frac{1}{2} \xi_{0}\right)-\sin \frac{1}{2} \xi_{0}} .
$$

Making use of a standard formula in the theory of elliptic integrals, the solution for $\phi$ can be expressed in terms of the Jacobian elliptic integral. Thus

$$
\pm \phi=\frac{1}{\sqrt{\triangle}} \int^{\psi} \frac{d \varsigma}{\sqrt{1-k^{2} \sin ^{2} \varsigma}}
$$

where

$$
k^{2}=\frac{1-\sin \frac{1}{2} \xi_{0}}{2},
$$

and

$$
\sin ^{2} \psi=\frac{1-\sin \left(\xi-\frac{1}{2} \xi_{0}\right)}{1-\sin \frac{1}{2} \xi_{0}}
$$

From equation (88) and (93), it follows that $\sin ^{2} \psi=$ 1 both when $\xi=\xi_{0}$ (at aphelion) and $\xi=\pi$ (at the singularity). Moreover, $\sin ^{2} \psi=0$ when $\xi=\tan ^{-1} \frac{2 \mu e}{6 \mu+1}$. Therefore, $\psi$ takes the value zero within the range (86) of $\xi$. We conclude that the range of $\psi$ associated with the range of $\xi$ is

$$
-\frac{\pi}{2} \leq \psi \leq \frac{\pi}{2} \quad\left(\xi_{0} \leq \xi \leq \pi\right) .
$$

Accordingly, we may write the solution for $\phi$ as

$$
\phi=\frac{1}{\sqrt{\triangle}}[K(k)-F(\psi, k)]
$$

where we have assumed that $\phi=0$ at the singularity where $\xi=\pi$ and $\psi=\pi / 2$.

\section{The Cardioid type Geodesic}

For this case, we shall must consider that $r_{+} \leq r \leq r_{0}$, and also we suppose that the aphelion distance is $r_{0}=$ $2 M$, this mean that $E^{2}=\frac{L^{2}}{\ell^{2}}=E_{\Lambda}^{2}-1$, and therefore

$$
\dot{r}^{2}=\frac{L^{2}}{r^{3}}(2 M-r), \quad r<2 M .
$$

Dividing the above equation with (11), we obtain

$$
\left(\frac{d r}{d \phi}\right)^{2}=r(2 M-r)
$$


An elemental integration of the last equation yield

$$
r(\phi)=M(1+\cos \phi)
$$

which is the equation of the cardioid. A surprising result is the independence of the angular momentum for the trajectory of non radial photons in this region. There is only a dependence on the Black Hole mass. This trajectory is showed in the (FIG 6)

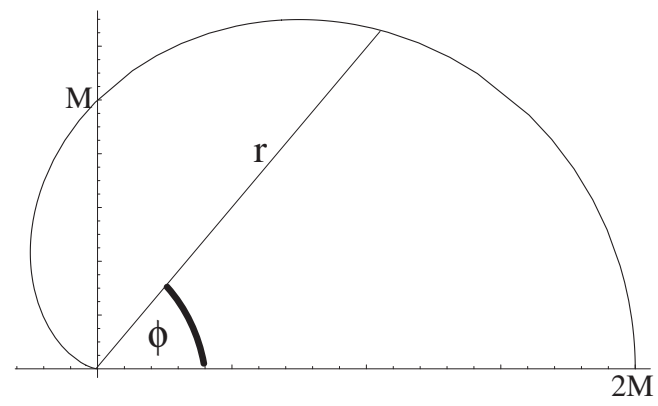

FIG. 6: The figure shows the cardioid for non-radial photons. The trajectory is independent on the angular momentum of the particles and only depend on the mass of black hole.

In accordance with our suggest that the photon start from $r_{0}=2 M$, we can say that the photon crosses the event horizon for an angle $0<\phi_{+}<\pi$.

On the other side, replacing (98) in (11) one get

$$
M^{2}(1+\cos \phi)^{2} \frac{d \phi}{d \tau}=L
$$

After a elemental integration, this last equation becomes

$$
\tau(\phi)=\frac{M^{2}}{2 L}[3 \phi+(4+\cos \phi) \sin \phi] .
$$

inverting (98) and inserting the result in (100) we obtain

$$
\tau(r)=\frac{M^{2}}{2 L}\left[3 \cos ^{-1}\left(\frac{r}{M}-1\right)+\left(3+\frac{r}{M}\right) \sqrt{2-\frac{r}{M}}\right] .
$$

\section{The unbound geodesics}

\section{The Critical Orbits}

Returning to the equation (70), we first consider the different cases that must be distinguished. They are related with the disposition of the roots of the cubic equation

$$
z(u)=2 M u^{3}-u^{2}+\frac{1}{D^{2}}=0 .
$$

The sum and the products of the roots $u_{1}, u_{2}$, and $u_{3}$ of this equation are given (see appendix $\mathrm{B}$ for more details)

$$
u_{1}+u_{2}+u_{3}=\frac{1}{2 M} \quad \text { and } \quad u_{1} u_{2} u_{3}=-\frac{1}{2 M D^{2}} .
$$

Clearly the equation $z(u)=0$ must allow a negative real root; and the two remaining roots may either be real (distinct or coincident) or be a complex conjugate pair. The case when two of the positive roots coincide plays a specially decisive role in the discrimination of the null geodesics. Thus, we shall consider this critical case first. The derivative of equation (102), namely,

$$
z^{\prime}(u)=6 M u^{2}-2 u=0,
$$

allows $u=(3 M)^{-1}$ as a root; and $u=(3 M)^{-1}$ will be a root of equation (102) (indeed, a double root) if

$$
D^{2}=27 M^{2} \quad \text { or } \quad D=3 \sqrt{3} M .
$$

From the condition on the product of the roots, we infer that the roots of $z(u)=0$ are

$$
u_{1}=-\frac{1}{6 M} \quad \text { and } \quad u_{2}=u_{3}=\frac{1}{3 M} .
$$

Therefore, [69) can be rewritten as

$$
\left(\frac{d u}{d \phi}\right)^{2}=2 M\left(u+\frac{1}{6 M}\right)\left(u-\frac{1}{3 M}\right)^{2} .
$$

This equation is satisfied by the substitution

$$
\frac{1}{u}=r=\frac{3 M}{\frac{3}{2} \tanh ^{2} \frac{1}{2}\left(\phi-\phi_{0}\right)-\frac{1}{2}},
$$

where $\phi_{0}$ is a constant of integration. If $\phi_{0}$ is chosen to be

$$
\tanh ^{2} \frac{\phi_{0}}{2}=\frac{1}{3}
$$

then

$$
u=0 \quad \text { and } \quad r \rightarrow \infty \quad \text { when } \quad \phi=0 .
$$

But we also notice that

$$
u=\frac{1}{3 M} \quad \text { when } \quad \phi \rightarrow \infty .
$$

Therefore, a null geodesic arriving from infinite with an impact parameter $D=3 \sqrt{3} M$ approaches the circle of radius $3 M$, asymptotically, by spiraling around it. 


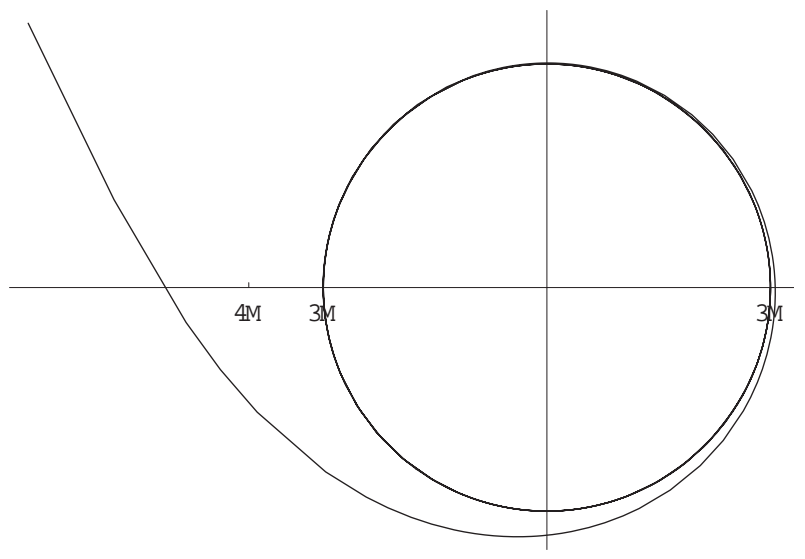

FIG. 7: The polar plot shows the critical null geodesic. The photons arriving from infinite and approaches the circle of radius $3 M$, asymptotically, by spiraling around it.

Associated with the orbit (102), we must have an orbit of the second kind which, originating at the singularity, approaches, from the opposite side, the same circle at $r=3 M$, asymptotically, by spiralling around it. Such an orbit can be obtained by the substitution

$$
u=\frac{1}{3 M}+\frac{1}{2 M} \tan ^{2} \frac{1}{2} \xi
$$

in (102). Then, after a little manipulation it reduce to

$$
\left(\frac{d \xi}{d \phi}\right)^{2}=\sin ^{2} \frac{1}{2} \xi
$$

Thus, this last equation is integrating, yields

$$
\phi=2 \ln \left(\tan \frac{1}{4} \xi\right) \quad \text { or } \quad \tan \frac{1}{4} \xi=e^{\phi / 2} .
$$

inserting this result in (112), we obtain

$$
\frac{1}{r}=u=\frac{1}{3 M}+\frac{2 e^{\phi}}{M\left(e^{\phi}-1\right)^{2}}
$$

along this orbit

$$
\begin{aligned}
& u \rightarrow \infty \quad \text { and } \quad r=0 \text { when } \quad \phi \rightarrow 0 \\
& \text { and } \quad u=\frac{1}{3 M} \quad \text { as } \quad \phi \rightarrow \infty \text {. }
\end{aligned}
$$

The above solution with the sign of $\phi$ reversed may be considered as a "continuation" of the solution (108).

\section{(i) The cone of avoidance}

At any point we can define a cone of avoidance whose generators are the null rays, described by the solution

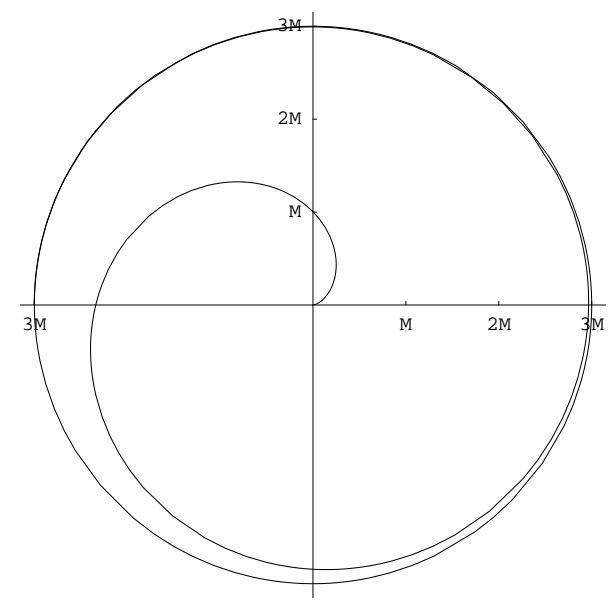

FIG. 8: The polar plot shows the critical null geodesic of the second kind. This orbit may be considered as a continuation of the critical orbit showed in FIG 7

(108) of equation (107), passing through that point, since, as is clear on general grounds and as we shall establish analytically in the Orbits With Imaginary Eccentricities below, light rays, included in the cone, must necessarily cross the horizon and get trapped.

If $\Psi$ denotes the half-angle of the cone (directed inward at large distances), then

$$
\cot \Psi=+\frac{1}{r} \frac{d \tilde{r}}{d \phi}
$$

where

$$
d \tilde{r}=\frac{1}{\sqrt{1-\frac{2 M}{r}+\frac{r^{2}}{\ell^{2}}}} d r,
$$

is an element of proper length along the generators of the cone. Therefore,

$$
\begin{aligned}
\cot \Psi & =+\frac{1}{r \sqrt{1-\frac{2 M}{r}+\frac{r^{2}}{\ell^{2}}} \frac{d r}{d \phi}} \\
& =-\frac{1}{\sqrt{2 M} \sqrt{\frac{1}{2 M \ell^{2}}+\frac{u^{2}}{2 M}-u^{3}}} \frac{d u}{d \phi},
\end{aligned}
$$

where $u=1 / r$. In equation (119) we may substitute for $d u / d \phi$ from equation (107). In this way we obtain

$$
\cot \Psi=\frac{\left(\frac{r}{3 M}-1\right) \sqrt{\frac{r}{6 M}+1}}{\sqrt{\frac{r}{2 M}-1+\frac{r^{3}}{2 M \ell^{2}}}}
$$

or equivalently

$$
\tan \Psi=\frac{\sqrt{\frac{r}{2 M}-1+\frac{r^{3}}{2 M \ell^{2}}}}{\left(\frac{r}{3 M}-1\right) \sqrt{\frac{r}{6 M}+1}} .
$$




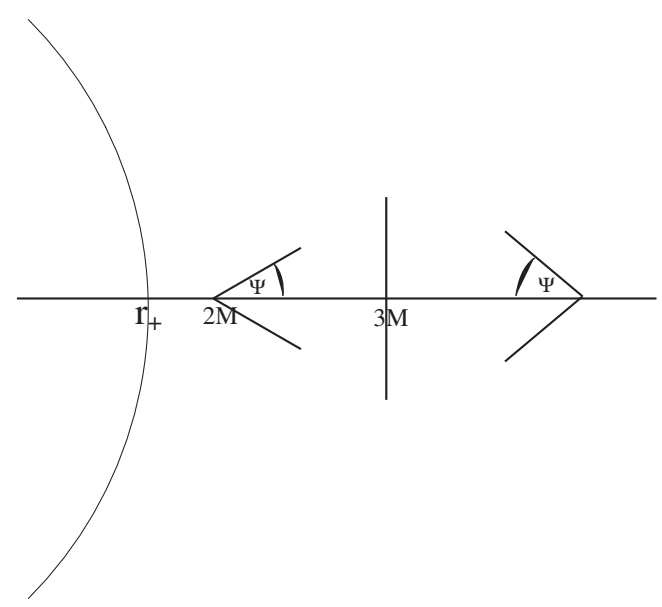

FIG. 9: The figure shows the cones of avoidance. Due to the inclusion of the negative cosmological constant, the event horizon appear in a distance lower than $2 M$ and consequently de cone of avoidance at these distance is not close as the Schwarzschild case.

From this last equation it follows that

$$
\begin{array}{rlrl}
\Psi \sim \frac{3 \sqrt{3} M}{r} \sqrt{1+\frac{r^{2}}{\ell^{2}}} & \text { as } & r \gg 1, \\
\Psi=\frac{\pi}{2} & \text { for } & r=3 M, \\
\text { and } & \Psi=0 & \text { for } & r=r_{+} .
\end{array}
$$

where we have assumed that for $r \gg 1, r^{2} / \ell^{2}<1$.

\section{Orbits Of The First Kind}

This case occur when all the roots of the cubic equation $z(u)=0$ are real and the two positive roots are distinct. Let the roots be

$u_{1}=\frac{P-2 M-Q}{4 M P}, \quad u_{2}=\frac{1}{P}, \quad u_{3}=\frac{P-2 M+Q}{4 M P}$.

Notice that the sum of the roots has been arranged to be equal to $1 / 2 M$ as it is required (see (B8) ), and $u_{1}<0$. Also, the ordering of the roots, $u_{1}<u_{2}<u_{3}$, requires that

$$
Q+P-6 M>0 .
$$

Evaluating

$$
z(u)=2 M\left(u-u_{1}\right)\left(u-u_{2}\right)\left(u-u_{3}\right),
$$

with $u_{1}, u_{2}$ and $u_{3}$ given in equation (123), and comparing the result with the expression (70), we obtain the relations

$$
Q^{2}=(P-2 M)(P+6 M),
$$

and

$$
\frac{1}{D^{2}}=\frac{1}{8 M P^{3}}\left[Q^{2}-(P-2 M)\right]
$$

Inserting the first of these relation into the second one get

$$
D^{2}=\frac{P^{3}}{P-2 M}
$$

On the other side, with the aid of (126), the inequality (124) gives

$$
(P-2 M)(P+6 M)>(P-6 M)^{2},
$$

or, after a little manipulation

$$
P>3 M \text {. }
$$

Using the substitution

$$
u-\frac{1}{P}=-\frac{Q-P+6 M}{8 M P}(1+\cos \chi),
$$

or, equivalently

$$
u+\frac{Q-P+2 M}{4 M P}=\frac{Q-P+6 M}{8 M P}(1-\cos \chi),
$$

we obtain

$$
\begin{array}{rrr}
u=\frac{1}{P} & \text { when } & \chi=\pi, \\
u=0 \quad \text { and } & r \rightarrow \infty & \text { when } \\
\sin ^{2} \frac{\chi}{2}=\frac{Q-P+2 M}{Q-P+6 M}=\sin ^{2} \frac{\chi_{\infty}}{2}, & \text { say }
\end{array}
$$

substituting (131) and (132) in (70) we obtain

$$
\left(\frac{d \chi}{d \phi}\right)^{2}=\frac{Q}{P}\left(1-k^{2} \sin ^{2} \frac{\chi}{2}\right),
$$

where

$$
k^{2}=\frac{Q-P+6 M}{2 Q} .
$$

In terms of Jacobi elliptic integrals, our solution can be write as

$$
\phi=\frac{2 P}{Q}\left[K(k)-F\left(\frac{\chi}{2}, k\right)\right] .
$$

It is possible, in this case, return to original variable $r$. In fact, using (132) in the above equation, we get 


$$
r(\phi)=\frac{4 M P}{4 M s n^{2}\left(K(k)-\frac{Q}{2 P} \phi\right)-(Q-P+2 M) c n^{2}\left(K(k)-\frac{Q}{2 P} \phi\right)} .
$$

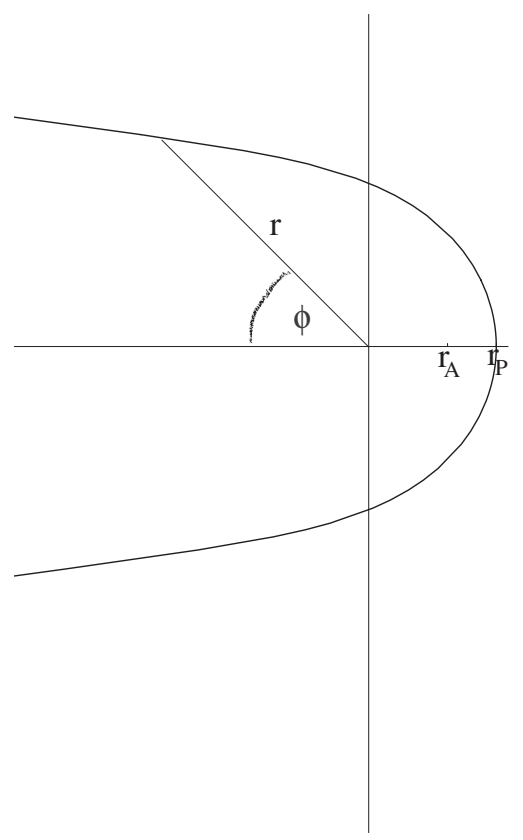

FIG. 10: The polar plot shows the null geodesic of the first kind. This orbit correspond to the motion of photon, which posses energy $E_{0}^{2}$, such that $E_{\Lambda}^{2}-1<E_{0}^{2}<E_{C}^{2}$. The motion is unbounded and the photon has a perihelion distance, $r=P$.

\section{Orbits Of The Second Kind}

In this case the physical range is $u>u_{3}>u_{2}$. With the substitution

$$
u=\frac{1}{P}+\frac{Q+P-6 M}{4 M P} \sec ^{2} \frac{\chi}{2},
$$

we obtain

$$
\begin{array}{rlr}
\text { aphelion } \quad u=u_{3}=\frac{Q-P-2 M}{4 M P} \text { and } \quad \chi=0 \\
u \rightarrow \infty \quad \text { and } \quad r \rightarrow 0 \quad \text { when } \quad \chi=\pi .
\end{array}
$$

Replacing (138) in (70) one get that its reduce to the same form (134) and with the same value of $k^{2}$; and we may now write
Therefore, one get

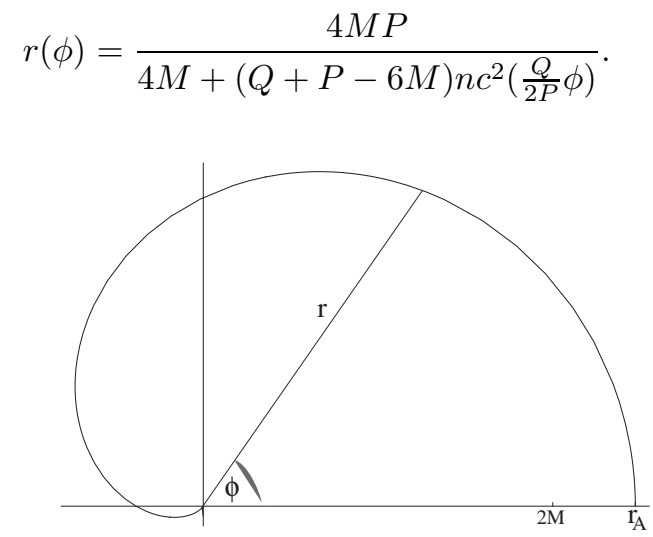

FIG. 11: The polar plot shows the null geodesic of the second kind. This orbit correspond to the motion of photon, which posses energy $E_{0}^{2}$, such that $E_{\Lambda}^{2}-1<E_{0}^{2}<E_{C}^{2}$. It are falling from an aphelion distance, $r=A$, crossing the event horizon, $r_{+}<2 M$, and finally arrive to the singularity.

\section{Orbits With Imaginary Eccentricities}

Finally, we consider the case when two roots of the equation $z(u)=0$ are complex conjugate pair, i.e. $u_{2}=$ $u_{3}^{*}$, while the remained root has a negative value. We shall now write the roots in term of an imaginary eccentricities as

$$
u_{2}=\frac{1+i e}{R} \quad \text { and } \quad u_{3}=\frac{1-i e}{R} .
$$

in this way, using $(\underline{B})$ we get for the negative real valued root $u_{1}$

$$
u_{1}=\frac{1}{2 M}-2 R
$$

yielding to the following expression for $z(u)$

$$
z(u)=2 M\left(u-\frac{1}{2 M}+\frac{2}{R}\right)\left[\left(u-\frac{1}{R}\right)+\frac{e^{2}}{R^{2}}\right],
$$

The sum of products of the roots, (B9), and the product of the root, (B10), gives

$$
e^{2}=\frac{3 \mu-1}{\mu}
$$


and

$$
\frac{D^{2}}{M^{2}}=\frac{1}{\mu(4 \mu-1)^{2}}
$$

respectively. These relation requires

$$
\mu>\frac{1}{3} \quad \text { and } \quad D<3 \sqrt{3} M .
$$

The substitution is

$$
u=\frac{1+e \tan \frac{\xi}{2}}{R},
$$

and the range of $u$ is now

$$
\infty>u>u_{0}, \quad \pi>\xi>\xi_{0} .
$$

Since these orbits are unbounded, the solution for $\phi$ is given by the same equation (91), but now $e$ have another value. In particular

$$
\phi_{\infty}=\frac{1}{\sqrt{\triangle}}\left[K(k)-F\left(\psi_{\infty}, k\right)\right]
$$

where $\psi_{\infty}$ (on substituting for $e^{2}$ its present value $\frac{3 \mu-1}{\mu}$ ) is now given by

$$
\sin ^{2} \psi_{\infty}=\frac{\triangle+1}{\triangle+6 \mu-1},
$$

where it should remembered that $\mu>1 / 3$; and further that

$$
\triangle=\sqrt{48 \mu^{2}-16 \mu+1} .
$$

\section{CONCLUSION}

We have studied the geodesic structure of AdS black holes analyzing the behavior of null and time-like geodesic by means of the effective potential which appears in the radial equation of motion.

For radial time-like geodesics, the effective potential is unbounded since $\Lambda<0$ acts as an attractive force which increases with the distance to the horizon. Particles always plunges into the horizon from an upper distance determined by the constant of motion $E$, which means that all radial particles are confined.

For non-radial time-like geodesics bounds orbits are allowed if $L^{2}>11.25 M^{2}$. For $L^{2}=11.25 M^{2}$ there is an unstable circular orbits. For $L^{2}<11.25 M^{2}$ no bounded orbits are allowed.
We have explicitly evaluated the proper and coordinate time in terms of the radial coordinates for radial time-like geodesics. Due to the attractive nature of the negative cosmological constant, these times are lower than in the Schwarzschild case. Moreover, Due to the Schwarzschild case is possible have non-confined particles, this comparatione is valid when the confined particles are considered. In this range, we get that physical features are the same in both cases.

We found that if $E<1$ and $L^{2}>11.25 M^{2}$, exist the orbits of the first kind which represents bound geodesics, with two extreme values. Three classes of orbits can be identify in this case: planetary orbits, circular orbits and asymptotic orbits, which approaches to the circular orbits by spiraling around it. Orbits of the second kind posses the same energy that the orbits of the first kind, but exist the regions at the left hand side of the top of the effective potential.

In the planetary orbits described above, we found an expression to the advance of perihelion in term of an infinity serial including the cosmological constant contribution, which contrast with the works of G. Kraniotis and S. Whitehouse 7], where the evaluation was done by means of the genus 2 siegelsche modular forms and including the Mercury's data. Our advantage consist in recuperate the classical serial for the Schwarzschild case, founded firstly by Einstein, for vanished cosmological constant.

For radial null geodesics, we obtain that, in the proper time, the situation is the same as in the Schwarzschild case. For the coordinate time we obtain a term which represent the Schwarzschild solution, founded in [1]], plus the cosmological constant contribution.

The effective potential for non-radial null geodesics show that the bounded orbits are allows if the constants of motion satisfies the condition $E^{2}<L^{2} / \ell^{2}$. These type of orbit not exist in the Schwarzschild spacetime, and correspond to confined photons which posses a return distance and plunges to the event horizon. The analytic solution is expressed by mean of elliptic function of Jacobi. If $E^{2}=L^{2} / \ell^{2}$, the return distance is $r=2 M$, the event horizon of the Schwarzschild spacetime, which correspond to a new type the orbit no allowed before. The analytic solution correspond to the cardioid type geodesic. If $E^{2}>L^{2} / \ell^{2}$, the exterior AdS black hole spacetime have the same form that the Schwarzschild spacetime. The cosmological constant effects are showed by mean of the cone of avoidance, which show that the photons feel an attractive force and the spacetime result to be more greater than the Schwarzschild spacetime. The likeness of these spacetimes implies that the solutions have the same analytic form, as we was showed in this paper (Section III.c).

\section{Acknowledgments}

We acknowledge fruitful discussions with members of the GACG (www.gacg.cl), specially with S. Lepe and 
M. Valenzuela. Also, we acknowledge useful discussions with M. Plyushchay, A. Anabalón, E. Carquín and P. Landeros. (M.O) and (J.R.V) thank to USACH for hospitality. This work was supported by CONICYT through Grant FONDECYT No. 1040229 (NC).

\section{APPENDIX A: SOLUTION TO THE CUBIC EQUATION $x^{3}+a x-b=0$.}

We are interest in solve cubic equations of the form

$$
x^{3}+a x-b=0 .
$$

where $a>0$ and $b>0$ (see equation (3), for instance). For this purpose, we make the change of variable $x=$ $Z \sinh \theta$, and multiplying by a scalar $\alpha$, we obtain the following equation

$$
\alpha Z^{3} \sinh ^{3} \theta+\alpha a Z \sinh \theta-\alpha b=0 .
$$

Considering the hyperbolic identity

$$
4 \sinh ^{3}(\theta)+3 \sinh (\theta)-\sinh (3 \theta)=0 .
$$

and comparing (A2) with (A3), we obtain the following relations

$$
\alpha Z^{3}=4, \quad \alpha a Z=3, \quad \alpha b=\sinh (3 \theta) .
$$

Solving the above equation for $Z$ and $\theta$, we obtain

$$
\begin{gathered}
Z=2 \sqrt{\frac{a}{3}} \\
\theta=\frac{1}{3} \sinh ^{-1}\left(\frac{3 b}{2} \sqrt{\frac{3}{a^{3}}}\right)+\frac{2 \pi}{3} n i,
\end{gathered}
$$

where the period of the hyperbolic function is $2 \pi i$. Therefore, the roots of the cubic equation are obtain replacing $n=0,1,2$. Thus, the roots of (A1) are

$$
\begin{gathered}
x_{1}=2 \sqrt{\frac{a}{3}} \sinh \left[\frac{1}{3} \sinh ^{-1}\left(\frac{3 b}{2} \sqrt{\frac{3}{a^{3}}}\right)\right], \\
x_{2}=2 \sqrt{\frac{a}{3}} \sinh \left[\frac{1}{3} \sinh ^{-1}\left(\frac{3 b}{2} \sqrt{\frac{3}{a^{3}}}\right)+\frac{2 \pi}{3} i\right], \\
x_{3}=2 \sqrt{\frac{a}{3}} \sinh \left[\frac{1}{3} \sinh ^{-1}\left(\frac{3 b}{2} \sqrt{\frac{3}{a^{3}}}\right)+\frac{4 \pi}{3} i\right] .
\end{gathered}
$$

Thus, considering (3), the event horizon $r_{+}$is obtained like (4).

\section{APPENDIX B: ROOTS OF THE POLYNOMIALS $\mathrm{P}(u)$}

We are interest in rewrite 5 degrees polynomials from it standard form

$$
\mathrm{P}(u)=u^{5}-a u^{4}+b u^{3}-c u^{2}+d u-e,
$$

to it factorized form

$$
\mathrm{P}(u)=\left(u-u_{1}\right)\left(u-u_{2}\right)\left(u-u_{3}\right)\left(u-u_{4}\right)\left(u-u_{5}\right),
$$

where the $u_{i}(i=1, \ldots, 5)$ are the roots of $\mathrm{P}(u)$. This roots must satisfies the following relations

$$
u_{1}+u_{2}+u_{3}+u_{4}+u_{5}=a,
$$

$$
\begin{array}{r}
u_{1} u_{2}+u_{1} u_{3}+u_{1} u_{4}+u_{1} u_{5}+u_{2} u_{3}+u_{2} u_{4} \\
+u_{2} u_{5}+u_{3} u_{4}+u_{3} u_{5}+u_{4} u_{5}=b,
\end{array}
$$

$$
\begin{array}{r}
u_{1} u_{2} u_{3}+u_{1} u_{2} u_{4}+u_{1} u_{2} u_{5}+u_{1} u_{3} u_{4} \\
+u_{1} u_{3} u_{5}+u_{1} u_{4} u_{5}+u_{2} u_{3} u_{4}+u_{2} u_{4} u_{5} \\
+u_{2} u_{3} u_{5}+u_{3} u_{4} u_{5}=c
\end{array}
$$

$$
\begin{array}{r}
u_{1} u_{2} u_{3} u_{4}+u_{1} u_{2} u_{3} u_{5}+u_{1} u_{2} u_{4} u_{5} \\
+u_{1} u_{3} u_{4} u_{5}+u_{2} u_{3} u_{4} u_{5}=d
\end{array}
$$

$$
u_{1} u_{2} u_{3} u_{4} u_{5}=e .
$$

If we have a $\mathrm{n}$ degrees polynomial, with $n<5$, then the above formulas can be used. For example if $n=3$, implies that $d=e=0$ and $u_{4}=u_{5}=0$, therefore the two last equations, (B6) and (B7) are equals to zero (i.e., $\left.\mathrm{P}(u)=u^{2}\left(u^{3}-a u^{2}+b u-c\right) \equiv u^{2} \mathrm{~g}(u)\right)$. The remaining equations are

$$
\begin{gathered}
u_{1}+u_{2}+u_{3}=a, \\
u_{1} u_{2}+u_{1} u_{3}+u_{2} u_{3}=b, \\
u_{1} u_{2} u_{3}=c .
\end{gathered}
$$


[1] Perlmutter, S., et al. 1998, Nature, 391, 51.

[2] Peebles, P.J.E., 1998, astro-ph/9806201

[3] F. Kotler, Annalen Physik, 56, 410, 1918.

[4] M.J. Jaklitsch, C. Hellaby and D.R. Matravers, Gen. Rel. Grav. 21 (1989) 941.

[5] Z. Stuchlík, M. Calvani, Gen. Rel. Grav. 23, (1991) 507.

[6] J. Podolsky, Gen. Rel. Grav. 31, (1999) 1703.

[7] G. V. Kraniotis, S. B. Whitehouse, Classical Quantum Gravity 20, (2003) 4817-4835.

[8] G. V. Kraniotis, "Precise relativistic orbits in Kerr spacetime with a cosmological constant", arXiv:gr-qc/0405095

[9] R. Adler, M. Bazin and M. Schiffer, Introduction to general relativity, McGraw-Hill,1975.

[10] B. Shutz, A first course in general relativity, Cambridge university press, 1990.

[11] S. Chandrasekhar, The mathematical theory of black holes, Oxford university press, 1993.

[12] S. Cornbleet, Am. J. Phys 61 (7)(1993).

[13] J. N. Islam, Phys. Lett. A 97(1983).

[14] L. D. Landau and E. M. Lifshitz, Classical field theory , Pergamon press, London, 1959.

[15] C. W. Misner, K. S. Thorne and J. A. Wheeler, Gravitation, Freeman, San Francisco, 1973.

[16] L. Fidkowski, V. Hubeny, M. Kleban and S. Shenker, JHEP 0402, (2004) 014.

[17] K. Lake, Phys. Rev. D 65, 087301 (2002).

[18] W. H. C. Freire, V. B. Bezerra, J. A. S. Lima, Gen. Rel. Grav. 33, (2001) 1407.

[19] This mean that the solution of the elliptic curve cannot be a elliptic function. 